pentans mit einer Methyl- und Propenyl-Gruppe in der Seitenkette ist. Nach Bredt') wird dies in den beiden Formeln ausgedrückt:<smiles>CC(C)=C1[C@@H](C(=O)C(C)C)C[C@H]1C</smiles>

Nur die Stellung der doppelten Bindung blieb noch aufzuklären. Da nun Campherphoron mit Hydroxylamin ein wahres Hydroxylaminderivat und kein Oxim liefert, so ist festgestellt, dass sich die doppelte Bindung in $\alpha-\beta$-Stellung zum Carbonyl, und nicht, wie in Formel II, in $\beta-\gamma$-Stellung befinden kann. Zum Scbluss nachen wir noch darauf aufmerksam, dass die ${ }^{-}$Formeln der von Kerp seinerzeit hergestellten Derivate des Camphoronhydroxylamins einiger Revision bedürfen, so hat das durch Reduction erhaltene Amin nunmehr die Constitution

a. s. w.

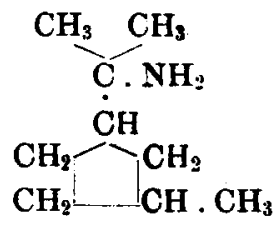

206. C. Harries und F. Mayrhofer: Ueber das Oxaminocarvoxim und seine Ueberführung in Dihydrocarvyldiamin.

(Eingegangen am 1. Mai.)

In einer kurzen Abhandlung ${ }^{2}$ ), Oxydation des Oxaminocarroximsk betitelt, ist bereits..eine Beschreibung der Bereitungsweise des Oxaminocarroxims gegeben.

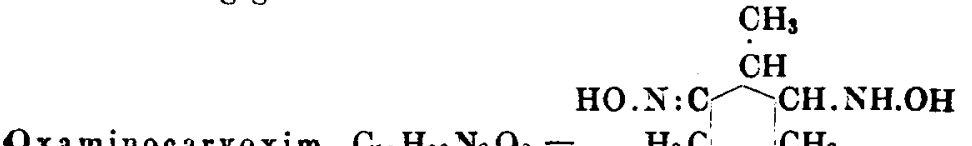

$$
\begin{aligned}
& \text { Oxaminocarroxim, } \mathrm{C}_{10} \mathrm{H}_{19} \mathrm{~N}_{2} \mathrm{O}_{2}= \\
& { }_{\mathrm{H}_{2} \mathrm{C}}^{\stackrel{\mathrm{CH}}{\mathrm{C}}{ }_{\mathrm{CH}_{3}}^{\mathrm{C}} \mathrm{CH}_{3}}
\end{aligned}
$$

Besser wird diese Verbindung nach folgendem Verfahren in reinem Zustand erhalten: $100 \mathrm{~g}$ Carrol werden mit einer aus $92.66 \mathrm{~g}$

1) Ann. d. Chem. 289, 9; a. a. Semmler, diese Berichte 25, 3520.

Diese Berichte 31, 1810. 
Hydrbxylaminchlorbydrat und $30.6 \mathrm{~g}$ Natrium gewonnenen methylalkoholischen Lösung von freiem Hydroxylamin in zusammen $1300 \mathrm{cem}$ Methylalkohol 8 Tage bei gewöhnlicher Temperatur in verschlossenem Gefäss stehen gelassen. Darauf wird der Methylalkohol im Vacuum bei $40^{\circ}$ Heizbadtemperatur möglichst vollständig abdestillirt. Der lichtgelbe, syrupöse Rückstand wird in $750 \mathrm{ccm}$ ahsolutem Aether aufgenommen, filtrirt und hierzu eine Auflösung von $50.8 \mathrm{~g}$ wasserfreier Oxalsäure in $500 \mathrm{ccm}$ absolutem Aether gegeben. Fast momentan erstarrt die ganze Masse zu einem weissen Krystallbrei. Die Ausbeute an diesem Oxalat beträgt ca. $170 \mathrm{~g}$. Zur weiteren Verarbeitung empfieblt es sich nicht, dasselbe zu trocknen, weil es dann schwer löslich wird: Man nimmt es vielmehr im feuchten Zustande in $11 / 2 \mathrm{~L}$ Wasser ron höchstens $40^{\circ}$ auf und salzt die Base mit $1 \mathrm{~kg}$ Kaliumcarbonat. aus. Dieselbe wird mit Aether ausgeschüttelt und hinterbleibt nach dem Verdunsten desselben als lichtgelber Syrup (Ausbeute ca. $100-110 \mathrm{~g}$ ), der danu in kaltem Wasser schwer löslich wird; $1 \mathrm{~g}$ wird voll $20 \mathrm{ccm}$ bei Siedehitze aufgenommen. Bei der Darstellung des Oxaminoxims aus dem Oxalat hat man besonders darauf zu achten, dass das Oxalat in wässriger Lösung nicht zu stark erwärmt wird, da sich dasselbe sonst in Carroxim und $\mathrm{Hydr}$ oxylamin unter Bildung anderer unerfreulicher Syrupe zersetzt. Wird das unter den angegebenen Cautelen bereitete lichtgelbe Product im Vacuumexsiccator getrocknet, so erstart es allmählich im Verlauf von einigen Tagen ganz und gar zu einer weissen, aus undeutlich ausgebildeten, verfilzten Nädelchen oder Drusen bestehenden harten Krystallmasse. An der Luft wird dieselbe schnell schmierig. Der Schmelzpunkt liegt etwas undeutlich bei $60-65^{\circ}$, ein anderes Präparat gab den Schmp. $83-84^{\circ}$, wurde aber nur einmal erhalten. Es ist in Benzol, Alkohol, Aether leicht löslich und wird ron verdünnten Säuren und Alkalien spielend aufgenommen. Febling'sche Lösung wird schon in der Kälte stark r+ducirt. Die Analyse zeigt, dass ein reines Product vorliegt.

0.191 g Sbst.: $0.4244 \mathrm{~g} \mathrm{CO}_{2}$ und $0.1562 \mathrm{~g} \mathrm{H}_{2} \mathrm{O}$.

$0.2168 \mathrm{~g}$ Sbst.: $27.2 \mathrm{ccm} \mathrm{N}$ (21.in, 759.5 mm).

$$
\begin{aligned}
& \mathrm{C}_{10} \mathrm{H}_{18} \mathrm{~N}_{2} \mathrm{O}_{2} \text {. Ber. } \mathrm{C} \text { 60.66, H 9.03, N } 14.14 . \\
& \text { Gef. } 》 60.44, 》 9.06, 》 14.31 \text {. }
\end{aligned}
$$

In geringer Menge ist das Oxaminoxim unter vermindertem Druck unzersetzt destillirbar. In grösseren Quantitäten siedet es unter 6-7 mm Druck bei $190^{\circ}$, einige Tropfen gehen unzersetzt über und dann kocht die ganze-Masse explosionsartig auf, indem sie ein scliwarzes, colophoniumartiges Pech hiuterlässt.

Pikrat und Oxalat sind bereits früber beschrieben worden. Das Chlorhydrat wird als weisse, hygroskopische Masse erhalten durch 
Sättigen einerer ätherischen Lösung der Base mit Salzsäuregas in der Kälte.

Dibenzoyloxaminocarvoxim, $\mathrm{C}_{10} \mathrm{H}_{16} \mathrm{~N}_{8} \mathrm{O}_{2}\left(\mathrm{CO} \mathrm{C}_{6} \mathrm{H}_{5}\right)_{2}$, gewinnt man nach der Schotten-Baumann'schen Methode als weisses, undeutlich krystallinisches Pulver, welches mit Petroläther gewaschen und aus absolutem Alkohol umkrystallisirt - $1 \mathrm{~g}$ löst sich in $20 \mathrm{ccm}$ bei Siedehitze - bei $171-1720$ schmilzt.

$0.2134 \mathrm{~g}$ Sbst. (im Vacuum getr.): $0.5572 \mathrm{~g} \mathrm{CO}_{2}, 0.117 \pm \mathrm{g} \mathrm{H}_{2} \mathrm{O}$.

$0.1601 \mathrm{~g}$ Sbst.: $9.9 \mathrm{ccm} \mathrm{N}\left(19^{0}, 766.7 \mathrm{~mm}\right)$.

$$
\begin{aligned}
& \mathrm{C}_{24} \mathrm{H}_{26} \mathrm{~N}_{2} \mathrm{O}_{4} \text {. Ber. C 70.93, H 6.40, N 6.89. } \\
& \text { Gef. } 771.21 \text {, 6.11, } 7.20 \text {. }
\end{aligned}
$$

Diphenylcyanatverbindung, $\mathrm{C}_{10} \mathrm{H}_{16} \mathrm{~N}_{2} \mathrm{O}_{2}$ (CO . NH . $\left.\mathrm{C}_{6} \mathrm{H}_{5}\right)_{2}$. Durch Zusammenbringen aquimolekularer Mengen Base und Phenylcyanat in absolutem Aether nach Goldschmidt erhalten; krystallisirt in warzenförmigen Drusen, welche, aus Petrolüther oder Ligroïn gereinigt, bei $96-97^{\circ}$ schmelzen, indem sie schon bei $80^{\circ} \mathrm{zu}$ sintern anfangen.

$0.1674 \mathrm{~g}$ Sbst. (im Vacuum getr.): $0.406 \mathrm{~g} \mathrm{CO}_{2}$ und $0.0989 \mathrm{~g} \mathrm{H}_{2} \mathrm{O}$.

0.1343 g Sbst.: $15.5 \mathrm{ccm} \mathrm{N}\left(20^{\circ}, 755 \mathrm{~mm}\right)$.

$$
\begin{array}{r}
\mathrm{C}_{24} \mathrm{H}_{28} \mathrm{~N}_{4} \mathrm{O}_{4} \text {. Ber. C 66.05, H 6.42, N } 12.84 . \\
\text { Gef. 》 66.21, 6.56, 》 } 13.19 .
\end{array}
$$

Die Phenylsenfölverbindung krystallisirt aus. Methylalkohol in langen, prïchtigen Nadeln vom Schmp. 142-143 ${ }^{\circ}$.

\section{Oxydationsrersuche mit dem Oxaminooxim.}

Nachdem wir die Eigenschaften des wahren Oxaminocarroxims kennen gelernt hatten, lag es nahe, das Dihydroxylaminderivat, welches $O$. Wallach und Schrader ${ }^{1}$ ) früher in den Händen gehabt haben, damit zu vergleichen. Die genannten Forscher berichten darüber, "wenn die ätheralkoholische Mutterlauge von der Darstellung des Carroxims verdampft wird, hinterbleibt ein dicker Syrup, der beim Aufbewalıren zäher wird und eventuell nach monatelangem Stehen erhärtet. In diesem Stadium mit kaltem Aether übergossen, wird die Masse ron diesem Lösungsmittel nur noch theilweise leicht gelöst. Es bleibt ein weisser Rückstand, der, mit sehr viel heissem Aether aufgenommen, bei langsamem Verdunsten desselben krystallinisch herauskommt. Beim Umkrystallisiren wurde ein zwischen $170-173^{\circ}$ schmelzendes Product erhalten; durch fortgesetztes Umkrystallisiren konnte der Schmelzpunkt auf $174-175^{\circ}$ erhöht werden*.

Folgende Analysen werden dafür angegeben.

$\mathrm{C}_{10} \mathrm{H}_{18} \mathrm{~N}_{2} \mathrm{O}_{2}$. Ber. $\mathrm{C} 60.56$, H 9.11 .

Gef. I. " fil.87, II. 61.97, III.60.399, J. * 8.94, II. 9.08, ITI. 8.74. N $14.1 \%$.

IV. $14.114 ;$ V. 14.65 .

1) Ann. d. Chem. 279, 368. 
Analyse III und IV stammen von dem bei $174-175^{\circ}$ schmelzeuden Product.

Wie man sieht, stimmen diese Analysen mit Ausnahme derjenigen des Stickstoffs weder unter einander noch gut mit den für $\mathrm{C}_{10} \mathrm{H}_{16} \mathrm{~N}_{2} \mathrm{O}_{2}$ berechneten Werthen überein. Ausserdem steht die Angabe im Widerspruch mit unserenErfahrungen, dass das Oxaminoxim in Aether schwer löslich ist und sich dadurch iøoliren lässt.

Wir glauben nun die Verbindungen, welche Wallach und Schrader in den Händen gehabt haben, für das Gemisch von Körpern erklären zu können, welehes durch Oxydation des Oxaminocarroxims durch den Sauerstoff der Luft entsteht und dessen schon früher (Diese Berichte 31, 1810) Erwähnung geschehen ist. Dasselbe kann durch Waschen mit Aether gereinigt werden, wie $W$ allach und Schrader angegeben haben. Wäbrend der gelbe Syrup beim Stehen an der Luft sich bräunt und langsam oxydirt, aber selbst nach längerer Zeit (vier bis sechs Wochen) noch Fehling'sche Lösung in der Kälte reducirt, wird die ätherische Lösung schon beim Stehen im nicht verschlossenen Gefüss in kurzer Zeit, nach 14 Tagen in beträchtlicher Mengr, in das Oxydationsgemisch verwandelt. Beschleunigen kann man dies, wenn man durch die Lösung Luft hindurchsaugt.

$10 \mathrm{~g}$ Oxaminoxim wurden in $500 \mathrm{~g}$ Aether gelöst und in der beschriebenen Weise behandelt. Nach vier Tagen konnten $6.3 \mathrm{~g}$ Oxydationsproduct durch Waschen mit Aether isolirt werden, welches zwischen $150-170^{\circ}$ schmilzt. Der Rückstand bestand aus einem dunkelgefärbten, fadenziehenden Oele, welches Fehling'sche Lösung nicht mehr reducirt.

Dies Verfahren haben wir auch im Grossen angewandt und bessere Ausbeuten erhalten, als weun wir nach der früheren Methode, Erhitzen der wässrigen Lösung des Oxaminoxims mit gelbem Quecksilberoxyd, arbeiteten.

Seinerzeit ist angegeben worden, dass das bei der Oxydation erhaltene Product ein Gemisch von mindestens zwei Körpern ist. Löst man die Rohsubstanz in absolutem Alkohol, so seheiden sich beim Erkalten schöne Krystalle ab, welche nach mebrfachem Umkrystallisiren ans dem gleichen Lösungemittel constant unter Zersetzung bei 193-1940 bei schnellem Erhitzen, bei langsamem Erwärmen bei $198^{\circ}$ schmelzen. $1 \mathrm{~g}$ der schräg abgeschnittenen Prismen wird von ca. $15-16 \mathrm{ccm}$ siedendem Alkohol aufgenonmen. Sie lösen sich leicht in verdünnten Alkalien und Sãuren und reduciren beim Erwärmen stark Fehling'sche Flüssigkeit. Beim Kochen mit rerdünnter Schwefelsäure wird alles Hydroxylamin abgespalten und das Diketon, welches früher besclirieben wurde, rom Schnelzpunkt $1 ! 4^{0}$, gebildet. In diesem Oxydationspro- 
duct vom Schmp. $193-194^{\circ}$, liegt das wahre Dioxim der folgenden Formel vor:<smiles>CC(C)[C@H]1C[C@@H](C(O)=NO)[C@H](C)[C@H]1NO</smiles>

$0.1914 \mathrm{~g}$ Sbst. (bei $100^{\circ}$ getrocknet): $0.4305 \mathrm{~g} \mathrm{CO}, 0.1405 \mathrm{~g} \mathrm{H}_{2} \mathrm{O}$.

$0.2028 \mathrm{~g}$ Sbst.: $25.4 \mathrm{~g} \mathrm{~N}\left(23.5^{\circ}, 760 \mathrm{~mm}\right)$.

$\mathrm{C}_{10} \mathrm{H}_{16} \mathrm{~N}_{2} \mathrm{O}_{2}$. Ber. C (11.22, $\mathrm{H} 8.16, \mathrm{~N} 14.29$.

Gef. \61.34, \$ 8.15 , 14.26 .

Dibenzoylkörper des Dioxims, $\mathrm{C}_{10} \mathrm{H}_{14}\left(\mathrm{~N} . \mathrm{O}_{2} \mathrm{C} . \mathrm{C}_{8} \mathrm{H}_{5}\right)_{2}$.

Nach dem Schotten-Baumann'schen Verfahren dargestellt; schmilzt, aus Benzol und Petrolather umkrystallisirt, bei $118-120^{\circ}$ unter Zersetzung.

$0.1486 \mathrm{~g}$ Sbst. (im Vacuum getr.): $0.3871 \mathrm{~g} \mathrm{CO}_{2}, 0.0798 \mathrm{~g} \mathrm{H}_{2} \mathrm{O}$.

$$
\begin{aligned}
& \mathrm{C}_{24} \mathrm{H}_{24} \mathrm{~N}_{2} \mathrm{O}_{4} \text {. Ber. C 71.28, H } 5.94 \text {. } \\
& \text { Gef. * 71.05, } 5.96 \text {. }
\end{aligned}
$$

Aus der Mutterlauge des Dioxins vom Schmp. $194^{\circ}$ erhält man einen anderen Körper, der undeutlich bei $153-155^{\circ}$ schmilzt. Derselbe, früher als Hauptproduct der Oxydation beschrieben, besitzt nach der Stickstoffbestimmung ebenfalls die Zusammensetzung $\mathrm{C}_{10} \mathrm{H}_{16} \mathrm{~N}_{2} \mathrm{O}_{2}$. Die Stickstoff bestimmungen sind aber hier nicht allein entscheidend; die Kohlenwasserstoffanalysen ergaben etwas abweichende Resultate, welche anzeigen, dass dieser Körper nicht isomer mit dem anderen ist. Da die Verbindung nur in geringerer Quantität, wie nachtráglich festgestellt wurde, auftritt, wurde sie nicht weiter untersucht. Hinzuzufügen ist noch, dass sie beim Erwărmen ebenfalls Fehling'sche Lösung reducirt.

Wir haben noch eine ganze Reihe ron Oxydationsversuchen mit dem Oxaminooxim angestellt:

Alkalische Ferricyankalilösung, berechnete Mengen chromsaures Kalium und Schwefelsäure, geben ebenfalls das Dioxingemisch, aber bedeutend unreiner, als nach dem oben mitgetheilten Verfahren; Fehling'sche Lösung in der Kälte tührt zu einer kupferbaltigen Substanz. Interessant ist das Verhalten von Permanganat. $5 \mathrm{~g}$ Oxaminooxim wurden in der gerade genũgenden Menge Natronlauge gelōst nnd dazu $2.65 \mathrm{~g}$ Kaliumpermanganat in $100 \mathrm{ccm}$ Wasser unter gater Kühlung gegeben. Nach dem Abfiltriren des Braunsteins wurde das weingelbe Filtrat mit 50-procentiger Essigsäure genau neutralisirt, wobei sich das Oxydationsproduct abschied. Dasselbe war Carvoxim rom Schmp. 
71. Durch Permanganat wird also die Hydroxylamingruppe einfach eliminirt. Die Oxydation mit Quecksilberoxyd ist früher beschrieben worden.

\section{Reduction des Oxaminocarroxims.}

$$
\begin{aligned}
& \mathrm{CH}_{3} \\
& \stackrel{\mathrm{C}}{ } \mathrm{H}
\end{aligned}
$$

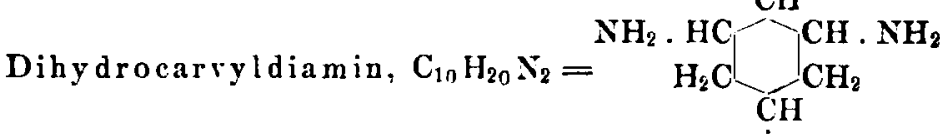

$$
\begin{aligned}
& \mathrm{H}_{2} \mathrm{C} \stackrel{\dot{\mathrm{C}}}{=} \mathrm{CH}_{3}
\end{aligned}
$$

$20 \mathrm{~g}$ Oxaminocarvoxim werdeu in $700 \mathrm{ccm}$ Alkohol gelïst und hierin $56 \mathrm{~g}$ Natrium eingetragen. Die Base wird mit Wasserdampf abgetrieben, die alkoholisch-wässrigen Filtrate genaı. mit verdūnnter Schwefelsäure neutralisirt und eingedampft. Aus dem Sulfat wird das Diamin mit starker Natronlauge in Freiheit gesetzt und mit Aether ausgeschüttelt. Nach dem Verdunsten des letzteren hinterbleiben $11.5 \mathrm{~g}$ eines dicken Oeles, welches zur Entfernung einer geringen Quantität Dihydrocarvylmonamin im Vacuum fractionirt wird. Die Hauptfraction $115-125^{\circ}$ bei $10 \mathrm{~mm}$ Druck enthält das Diamin. Zur weiteren Reinigung wird dasselbe in das saure Oxalat übergefährt. Eine absolut-ätherische Lösung der Base (11.j g) wird mit einer ebensolchen von wasserfreier Oxalsäure (ca. $13 \mathrm{~g}$ ) vermischt, es fällt sofort ein weisses Pulver aus, welches leicht von Wasser, schwer von Alkohol und Benzol aufgenommen wird. Der Schmelzpunkt liegt. bei $135-140^{\circ}$.

$0.1537 \mathrm{~g}$ Sbst. (im Vacuum getr.): $19.2703 \mathrm{~g} \mathrm{CO}_{2,}, 0.092 \mathrm{~g} \mathrm{H.2}$.

$$
\begin{aligned}
& \mathrm{C}_{14} \mathrm{~B}_{24} \mathrm{~N}_{2} \mathrm{O}_{8} \text {. Ber. C } 48.27 \text {, H } 6.89 \text {. } \\
& \text { Gef. » 47.96, \ } 7.17 \text {. }
\end{aligned}
$$

Aus dem Oxalat wird die Base wiederum durch Natronlauge in Freiheit gesetzt und mit festem Kali entwässert. Das Dihydrocarvyldiamin siedet bei gewöhnlichem Druck ron $2.58-260^{\circ}$ (Thermometer ganz im Dampf) oder bei $10 \mathrm{~mm}$ Druck von 122-1230 als eine wasserhelle, farblose, optisch inactive Flüssigkeit von basischem Geruch, welche aus der Luft begierig Kohlensäure unter Bildung eines ätherlöslichen, festen Carbaminates anzieht. Die Base ist in Wasser löslich und liefert nicht die Hofmann'sche Isonitrilreaction.

Das Dichlorhydrat wird erhalten als weisses, hygroskopisches Pulver durch Einleiten von wohlgetrocknetem Salzsäuregas in eine absolut-ätherische Lösung der Base $(1 \mathrm{~g}$ in $50 \mathrm{ccm}$ ) bis zur Sättigung. Die Ausbeute ist quantitativ.

$0.3282 \mathrm{~g}$ Sbst. (im Vacuum getr.): $0.3899 \mathrm{~g} \mathrm{AgCl}$.

0.2373 g Sbst.: $0.2850 \mathrm{~g} \mathrm{AgCl}$.

$$
\begin{aligned}
& \mathrm{C}_{16} \mathrm{H}_{20} \mathrm{~N}_{2} .2 \mathrm{HCl} \text {. Bir. Cl } 29.46 . \\
& \text { Gef. } \$ 24.34,29.70 .
\end{aligned}
$$


Das Chloroplatinat ist sebr leicht löslich in Wasser und undeutlich krystallinisch. Das Chloroaurat krystallisirt in etwas weniger löslichen, langen Nadeln.

Das Pikrat wird durch Zusammengeben einer ätherischen Lösung der Base mit einer solchen von Pikrinsäure in Aether-Alkohol gewonnen. Das gelbe Pulver wird ron Alkohol leicht aufgenommen and durch heisses Wasser zersetzt, es beginnt bei $229^{\circ}$ zu verkohlen und schäumt auf bei $250^{\circ}$.

$0.2069 \mathrm{~g}$ Sbst. (im Vacuum getr.): $0.3215 \mathrm{~g} \mathrm{CO}, 0.0817 \mathrm{~g} \mathrm{H}_{2} \mathrm{O}$.

0.1207 g Sbst.: $18.6 \mathrm{ccm} \mathrm{N}\left(18^{\circ}, 752 \mathrm{~mm}\right)$.

$$
\begin{aligned}
& \mathrm{C}_{22} \mathrm{H}_{26} \mathrm{~N}_{8} \mathrm{O}_{14} \text {. Ber. C 42.17, } \mathrm{H} 4.15, \mathrm{~N} 17.89 . \text {. } \\
& \text { Gef. "42.37, 》4.38, 》17.71. }
\end{aligned}
$$

Die Dibenzoylverbindung, nach der Schotten-Baumannschen Methode dargestellt, bildet, aus Alkohol umkrystallisirt, ein weisses Pulver rom Schmp. 275-276 ${ }^{\circ}$.

0.1532 g Sbst. (bei $110^{0}$ getrocknet): 0.4294 g $\mathrm{CO}_{2}, 0.105 \mathrm{~g} \cdot \mathrm{H}_{2} \mathrm{O}$.

$0.1902 \mathrm{~g}$ Sbst.: $12.6 \mathrm{ccm} \mathrm{N}\left(16^{0}, 751 \mathrm{~mm}\right)$.

$$
\begin{array}{ll}
\mathrm{C}_{24} \mathrm{H}_{28} \mathrm{~N}_{2} \mathrm{O}_{2} \text {. } & \text { Ber. C } 76.59, \mathrm{H} 7.44, \mathrm{~N} 7.44 . \\
& \text { Gef. 》 } 76.44 \text {, 》 7.61, 》 7.67. }
\end{array}
$$
Körper.

Als Nebenproduct beobachteten wir einen bei $123^{\circ}$ schmelzenden

$$
\begin{aligned}
& \text { Diphenyldihydrocarvyldiamindiharnstoff, } \\
& \left.\mathrm{C}_{10} \mathrm{H}_{16} \text { (NH . CO . NH . } \mathrm{C}_{6} \mathrm{H}_{5}\right)_{2} \text {. }
\end{aligned}
$$

Durch Zusammengeben berechneter Mengen Base und Phenylcyanat (3 Mol.-Gew.) in absolut-ätherischer Lösung gewonnen. Das aus verdünntem Alkohol umkrystallisirte Präparat schmilżt bei 214-2160.

$0.1898 \mathrm{~g}$ Sbst. (im Vicuum getr.): $0.4921 \mathrm{~g} \mathrm{CO}_{2}, 0.1300 \mathrm{~g} \mathrm{H}_{2} \mathrm{O}$.

$0.1433 \mathrm{~g}$ Sbst.: $16.9 \mathrm{ccm} \mathrm{N}(190,756 \mathrm{~mm})$.

$$
\begin{aligned}
& \mathrm{C}_{21} \mathrm{H}_{30} \mathrm{~N}_{4} \mathrm{O}_{4} \text {. Ber. C 70.93, H 7.38, N 13.79. } \\
& \text { Gef. \70.71, 》7.61, 》 } 13.56 \text {. }
\end{aligned}
$$

Diphenyldibydrocarvyldiamindithioharnstoff,

$$
\mathrm{C}_{10} \mathrm{H}_{16}\left(\mathrm{NH} \text {. CS . NH . } \mathrm{C}_{6} \mathrm{H}_{5}\right)_{2} \text {. }
$$

Durch Zusaminengeben berechneter Mengen Base und Phenylsenföl (2 Mol.-Gew.) in Methylalkohol erhalten und mit Aether gewaschen, schmilzt der Thioharnstoff bei $179-180^{\circ}$; er ist schwer löslich in Alkohol und Benzol.

$0.1628 \mathrm{~g}$ Sbst. (im Vacuum getr.): $0.3919 \mathrm{~g} \mathrm{CO}_{2}, 0.1039 \mathrm{~g} \mathrm{H}_{2} \mathrm{O}$.

$0.1716 \mathrm{~g}$ Sbst.: $18.9 \mathrm{ccm} \mathrm{N}\left(19^{\circ}, 7.55 \mathrm{~mm}\right)$.

$\mathrm{C}_{24} \mathrm{H}_{30} \mathrm{~N}_{4} \mathrm{~S}_{2}$. Ber. C 65.75, H 6.84, N 12.76.

$$
\text { Gef. " 65.66, ", 7.62, " } 12.65 \text {. }
$$


Bei der trocknen Destillation des Hydrochlorates sollte ein dem Cymol isomerer Kohlenwasserstoff entstehen:

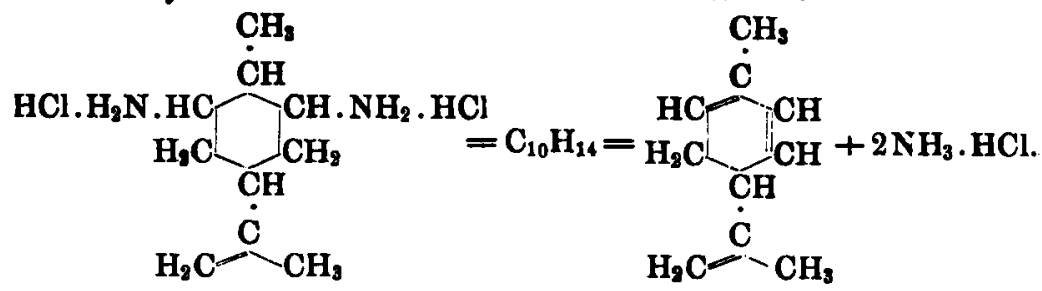

Wir unterwarfen etwa $30 \mathrm{~g}$ Hydrochlorat in Portionen je $5 \mathrm{~g}$ der trocknen Destillation. Dieselbe verläuft anscheinend ganz glatt, indem. weisser Salmiak als Rūckstand verbleibt und ein gelblicbes Oel übergeht. Dasselbe, ca. $8 \mathrm{~g}$, mit verdünnten Săuren durchgeschüttelt und getrocknet, sott bei ca. 170-175 , entfärbte Brom und Permanganatlösung, bestand aber zum grōsseren Theil aus Cymol. Wallach') hat bei der gleichen Destillation des Dihydrocarvylaminchlorhydrates Terpinen, $\mathrm{C}_{10} \mathrm{H}_{16}$, und ebenfalls Cymol erhalten. Wir baben vorlăufig die Untersuchung dieses interessanten Kohlenwasserstoffes, weil das Aasgangsmaterial in grösseren Mengen schwer zu beschaffen ist, aufgeben mūssen.

H. Goldschmidt ${ }^{2}$ ) hat gefunden, dass bei der Reduction des Carroxims mit Zinkstaub und Essigsäure zwei isomere Caroylamine entstehen, welche er durch die verschiedene Löslichkeit der Nitrate trennen konnte. Auch bei der Reduction des Oxamincarvoxims nit Natrium und Alkohol entsteht ein isomeres Basengemisch, welches man mittels der Nitrate scheiden kann. Das Diamin, welches sich als Hauptproduct bildet, ist oben beschrieben worden; über das beigemengte Isomere werden wir in Kürze berichten.

\section{A, Wohl und L. Neuberg: Ueber die Darstellung des Acroleïns.}

[Mittheilung aus dem I. Berliner chem. Universitats-Laboratorium.]

$$
\text { (Eingegangen am 28. April.) }
$$

Vor einiger Zeit hat A. Wohl die Synthese des r-Glycerinaldehydes vom Acroleïn aus beschrieben. Die Fortführung dieser Untersuchung, insbesondere die Versuche zur Spaltung des Glycerinaldehydes in seine stereoisomeren Componenten, erforderten grosse Mengen des Ausgangsmaterials, und es erschien deshalb wünschenswerth, ein bequemeres und ergiebigeres Darstellungsverfahren für Acroleïn aus-
1) Ann. d. Chem. 275, 125.
2) Diese Berichte 30, 2069. 